\title{
Wohnstandortgerechte städtische Mietzinse - eine Fallstudie Zürich
}

Wohnen als Funktion entspricht einem Elementarbedürfnis. Dieses Bedürfnis muss durch ein entsprechendes Wohnungsangebot gedeckt werden, welches den Nachfragerwünschen verschiedener Einkommensklassen gerecht wird. Die Qualität der angebotenen.Ware Wohnung widerspiege1t sich im Mietzins, welcher für eine nach Zimmerzahl abgegrenzte Wohneinheit von 3 Zimmern, je nach dem Wohnwert der angebotenen Wohnung, zwischen wenigen hundert und einigen tausend Franken variieren kann.

Der Mietzins wird nach marktwirtschaftlichen Gesichtspunkten, mehr oder weniger frei von rechtlichen Auflagen oder Bestimmungen, vom Eigentümer oder Verwalter festgesetzt. Und dabei kommt es bisweilen vor, dass qualitativ sich durchaus entsprechende Wohnungen mit bis $\mathrm{zu}$ $300 \%$ verschiedenen Mietzinsen gehandelt werden, obwohl kaum objektive Gründe für derart gravierende Abweichungen vorliegen.

$\mathrm{Zu}$ den rein marktwirtschaftlichen Kriterien für die Beurteilung eines Wohnwerts kommen seit geraumer Zeit immer neue Aspekte, vorab solche der Umweltqualität. Aus geographischer Sicht seien beispielsweise die Besonnung, die Dichte des umgebenden Grünraumes, die örtlichen lufthygienischen und akustischen Verhältnisse oder die verkehrsmässige Erschliessung des Wohnstandortes genannt. Dabei kann vermutet werden, dass diese nur erschwert messbaren qualitativen Faktoren vieles zu der "Grauzone" beitragen, als welche man die klaffenden, bisher unerklärten Mietzins-Disparitäten bezeichnen kann.

\section{Problemstellung und Forschungsziel}

Es drängte sich auf, in einem ersten Schritt die privatwirtschaftliche Mietzinskalkulation nach der Vollständigkeit möglicher Beeinflussungsfaktoren zu überprüfen. Dabei galt es herauszufinden, ob neben der traditionellen Berücksichtigung von Boden-, Bau- und Unterhaltskosten auch vorgehend erwähnte Umweltfaktoren in die Berechnung einfliessen und den Mietzins nach unten oder nach oben verändern.

In einem zweiten Schritt sollte versucht werden, das potentielle Gewicht der Umweltbedingungen für den Wohnwert abzugrenzen und zu überprüfen, wie sich diese im Mietzins niederschlagen. Und schliesslich wurde ein räumliches Verteilungsmuster von charakteristischen städtischen Wohnwertfaktoren angestrebt, welches zeigen sollte, dass die Hierarchie der berücksichtigten Faktoren quartierweise verschieden ist.

Befragungen bei rund 10 Immobilienverwaltungen haben ergeben, dass deren Mietzinskalkulationen lediglich auf streng messbaren und berechenbaren Grössen aufbauen. Qualitative Standortkriterien, welche zuerst mühevoll quantifiziert werden müssten, scheiden bei der Berücksichtigung häufig aus. Darauf wurde die folgende Arbeitshypothese formuliert, welche es mit einer Stichprobe aus städtischen Neubauwohnungen zu prüfen galt: 'Mietzins-Berechnungen für Neubauwohnungen stützen sich auf quantitative Kostengrössen unter Vernachlässigung von qualitativen Standortfaktoren". Als Rahmenbedingungen für diese Aussage wurden festgelegt: 1. Der Untersuchungsraum beschränkt sich auf die Stadt Zürich; 2. Es werden Neubauten zurück bis und mit Jahrgang 1963 berücksichtigt; 3 . Es werden nur nicht-subventionierte Wohnungen erfasst.

\section{Methodische Ueberlegungen}

Die Stadt Zürich wies im Jahr 1976 rund doppelt soviele 3-Zimmerwohnungen wie 2- oder 4-Zimmerwohnungen auf. Um den Untersuchungsprozess $\mathrm{zu}$ vereinfachen, wurde deshalb nur diese wichtigste Gruppe der 3- und 3y2-Zimmerwohnungen berücksichtigt, beschränkt auf den versorgungs-, erholungsund steuermässig in etwa homogenen städtischen Raum ohne Agglomerationsgemeinden.

Es konnte, sozusagen als Momentaufnahme, nur die statische Seite der Mietzins-Disparitäten erfasst werden. Dynamische Mietschwankungen (auf Hypothekarzinsveränderungen oder baulichen Wohnwertverbesserungen beruhend) wurden dagegen nicht mitberücksichtigt.

Mit der Tatsache, dass rund 5000 Einzelinformationen miteinander zu korrelieren seien, drängte sich die statistische Auswertung mithilfe einer schrittweisen Regression auf. Dadurch konnten sowoh1 einfache Abhängigkeiten zum Mietzins dargeste11t wie auch Gleichungen gesucht werden, in welchen sich der Mietzins mit den höchstkorrelierenden Variablen optimal erklären liess. Die einfachen Abhängigkeiten sollten also darüber Aufschluss geben, ob ein vermutetes Einflussmerkmal überhaupt mit dem Mietzins signifikant in Ver-

Martin Sommer, dipl. Geogr., Geographisches Institut der Universität Zürich, Blümlisalpstrasse 10, 8006 Zürich. 


\section{Abb. 1: DIE MIETZINSKALKULATION IM PRIVATEN WOHNUNGSBAU}

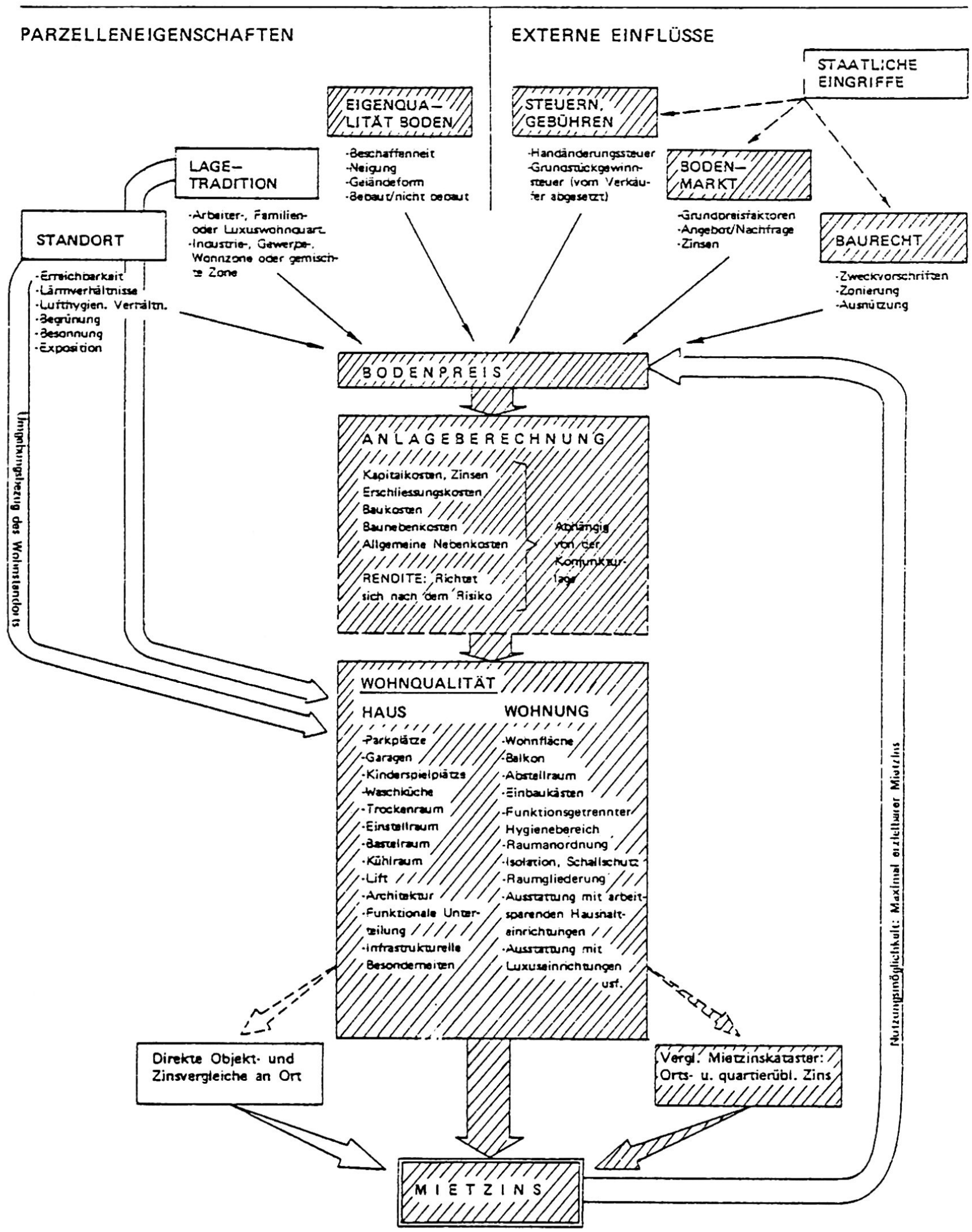

Entwurf: M. Sommer, 1978 
bindung gebracht werden kann. Und die schrittweise Regression sollte dann die Reihenfolge abnehmender Bedeutung abklären, welche den ausgewählten brauchbaren Erklärungsmerkmalen zukommt.

Bei der Variablenevaluation wurde eine operationable Höchstgrenze von 30 Variablen festgesetzt. Die mittels Interview-Technik durchgeführten Erhebungen von möglichen mietzinsrelevanten Merkmalen bei verschiedenen Verwaltungen und Hausbesitzern wurden durch solche Merkmale ergänzt, welche in Wohnungsannoncen hervorgehoben waren. Ausserdem wurde ein vom Bund erstelltes Wohnungsbewertungssystem (WBS) (1) zur Vervollständigung beigezogen. So ergaben sich schliesslich rund 200 feindifferenzierte Merkmale. Diese wurden dann zu 29 gröberen Beeinflussungsfaktoren vereinfacht und zusammengezogen. Die Abb.1 gibt eine Uebersicht über potentiell mögliche Einflussmerkmale auf den Mietwert. gemäss den Resultaten genannter Erhebungen.

\section{Kalkulationsschema für Mietzinse (Abb.1)}

Ueblicherweise folgt der Mietzinskalkulator den Pfeilen zwischen den schraffierten Merkmalspaketen. Die sogenannte Lagetradition und die umweltbedingten Standortfaktoren scheinen nur eine untergeordnete Bedeutung zu haben. Häufig genügen sogar eine Anlageberechnung und ein flüchtiger Vergleich mit dem Mietzinskataster sowie den marktüblichen Höchstmieten für entsprechende Objekte, um zu einem Mietwert zu gelangen. Sovie1 zeigen zusätzliche Ergebnisse aus derselben Interview-Umfrage, welche zur Erhebung potentie1ler Einflussmerkmale stichprobenartig durchgeführt wurde.

Eine zweite schriftliche Umfrage sollte nun genaue vergleichbare Daten zu den Anlagekosten, der Haus- und Wohnungsqualität sowie zu der Standortattraktivität einer Wohnung ergeben. Die Umfrage wurde auf breiter Basis angelegt ( 815 verschickte Fragebogen ), und einwandfrei ausgefüllt und wieder eingegangen waren schliesslich $15 \%$ aller Fragebogen. Im folgenden sollen die darin erfragten geographischen Standortfaktoren näher erläutert werden.

\section{Die geographischen Wohnstandortfaktoren}

Vorerst musste abgeklärt werden, in welcher Differenzierung die verschiedenen Variablen verarbeitbar erhä1tlich sind. So war es unmöglich, feindifferenzierte Lärmstördaten über den Strassen-, Schienen- und Flugverkehr an einem Wohnort $\mathrm{zu}$ erhalten. Lärmemissionsmessungen von Industriebetrieben liegen ebenfalls nicht vor, und Kindergeschrei wird individuell verschieden als Lärmquelle gedeutet oder als positives Wohnwertkriterium empfunden. Probleme ergaben sich aber gerade dadurch, dass einzelne Wohnstandorte nicht genügend genau in ihrer individuellen Ausprägung erfasst wurden und deshalb häufig etwas verallgemeinert charakterisiert werden mussten. Schiliesslich sind die folgenden Bewertungskriterien ausgewäh1t und zusammengestellt worden:
- Besonnung: Gutersohn (1934) (5) ermittelte quartierweise die maximal mögliche mittlere tägliche Sonneneinstrahlung. Mit der gleichen Methode wurden die fehlenden Werte für die damals noch nicht eingemeindeten Quartiere berechnet.

- Grünwert: Es wurde ein Naherholungs- oder Grünfaktor errechnet, welcher aus dem statistischen Anteil der Freihaltezonen und dem Waldflächenanteil an der Quartierfläche zusammengesetzt ist.

- Belichtung: Die Hauptexposition des Wohnzimmers sollte als Parameter für die natürliche Belichtung einer Wohnung dienen. Dabei waren nordbis ost- und südostorientierte Wohnungen selten zu finden (Abb.2). Südwestexponiert waren hingegen rund $37 \%$ aller untersuchten Wohnungen.

- Lufthygienische Verhä1tnisse: Der Schadstoffgehalt der Luft ist ebenfalls nur in Quartierraster-Messwerten greifbar. So liegt ein $\mathrm{SO}_{2}-$ Belastungskataster vor, welcher den gestellten Anforderungen genügen konnte.

- Beziehungspotential zum Geschäftszentrum (City): Sowoh1 für die Erschliessung mit öffentlichen wie mit privaten Verkehrsmitteln konnte je eine Erreichbarkeitsvariable geschaffen werden. Ein von der Stadt Zürich beauftragtes Ingenieurbüro ste11te freundlicherweise die Ergebnisse einer Untersuchung über feingliedrige Erschliessungspotentiale in der Stadt Zürich zur Verfügung.

- Lärmbelastung: Differenzierte Messresultate lagen nur lückenhaft vor. Eine näherungsweise Berechnung der Strassenlärmbelastung aus dem Verkehrsvolumen konnte ebenfalls nicht berücksichtigt werden, weil nur unvollständige Verkehrsfrequenz-Zählungen erhältlich waren. Dazu kommt, dass akustische Störungen von Wohnung zu Wohnung stark voneinander abweichen können, so dass die Messbarkeit einer durchschnittlichen Strassenlärmbelastung in Frage gestellt werden muss. Somit musste diese Variable ausgeschieden werden.

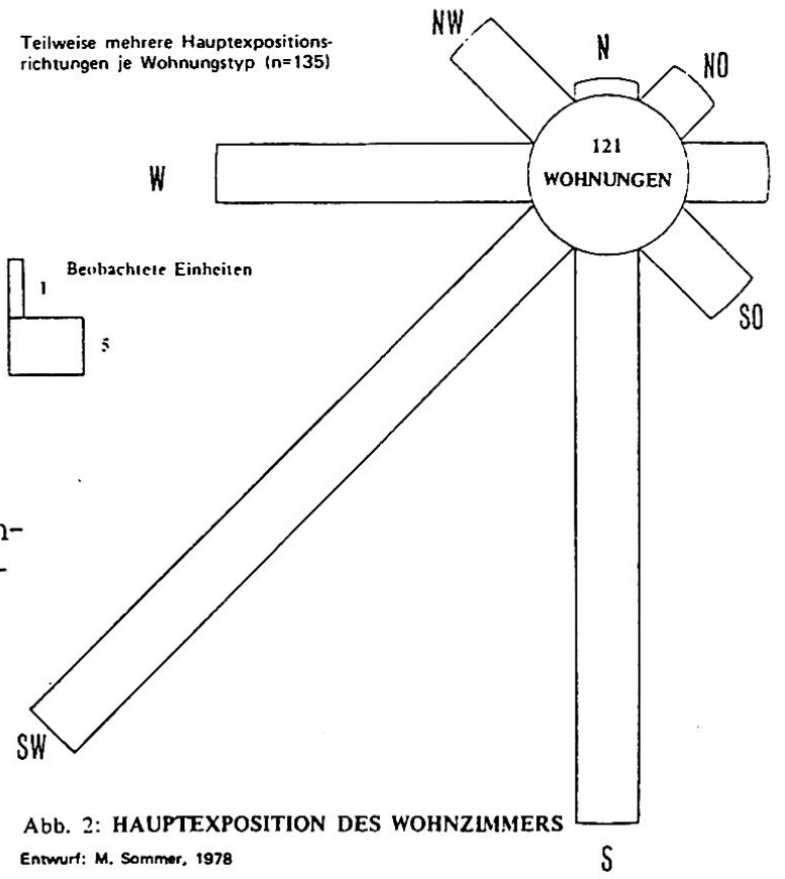


Im übrigen wurden neben den geographischen Wohnstandortskriterien eine Reihe von Kostenfaktoren und Wohnqualitätsfaktoren erfragt, um die kostenmässigen Kalkulationsgrundlagen und den Ausbaustandard der untersuchten Wohnungen zu erfassen. Erhoben wurden der Bodenpreis, die Baukosten inkl. Nebenkosten und die Bruttorendite, sowie die Summe der jährlichen Mieteinnahmen aus dem untersuchten objekt. Gesamthaft gingen aber nur wenige Zahlen ein.

Schwierigkeiten ergaben sich dabei insofern,als ein grosser Teil der angefragten Hausbesitzer keine Auskunft über die Bruttorendite ihres Objektes geben wollten. Zusätzliche Probleme bereitete schliesslich auch die Erhebung der Bodenpreise auf statistischer Basis, da Mittelwerte für ein ganzes Quartier im Individualfall zu ungenau sind und die $\mathrm{m}^{2}$-Preise aus den Steuerdossiers der verschiedenen Berechnungsansätze wegen nicht miteinander vergleichbar waren. Denn der Bodenpreis basiert auf unterschiedlichen Berechnungsgrundlagen, je nachdem, ob die Liegenschaft frei auf dem Markt gehandelt, vererbt oder "verschenkt"(d.h.zu einem symbolischen Preis abgesetzt) wurde.

\section{Gehobener Wohnkomfort durch Luxuseinrichtungen}

Unter Luxusfaktoren sollen im folgenden Wohnwertfaktoren höherer Ordnung verstanden werden, welche zu einer Anhebung des mittleren Wohnkomforts beitragen. Als solchen bezeichnet man das Vorhandensein der Grundinstallationen wie Heizung, elektrische Versorgung aller Zimmer, fliessendes kaltes und warmes Wasser, sowie die Ausstattung mit Bad und WC, Kochherd mit Normalbackofen, Kühlschrank, Küchenschrank, Spülkombination und Balkon.

Dabei sind $41 \%$ der untersuchten Wohnungen mit Spannteppichen ausgerüstet, und etwa $36 \%$ der Wohnungen verfügen über eine Geschirrwaschmaschine. Knapp 22\% sind mit einem Tiefkühlapparat ausgestattet, und rund 17\% der Backöfen wurden hochliegend montiert. Diese Spezialeinrichtung erleichtert zwar die Hausarbeit, erfordert aber den Einbau einer kompletten Küchen-Kombinationswand. Schliesslich verfügen knapp $10 \%$ der neuen 3-Zimmerwohnungen über ein Cheminée, und in $6 \%$ der
Fälle steht neben der Grundausrüstung mit einem Bad noch eine zusätzliche Dusche zur Verfügung. In keiner der untersuchten Wohnungen war aber eine wohnungseigene Waschmaschine zu finden. Durchschnittlich ist also jede funfte neue 3-Zimmerwohnung mit einem oder mẹreren Luxusfaktoren ausgestattet.

Die schrittweise Regression berücksichtigte nun der Reihe nach die folgenden Variablen (siehe Tabelle 1).

Mit den ausgewählten neun Variablen konnten so insgesamt $86 \%$ der aufgetretenen Wertestreuung der Bruttomietzinse erklärt werden. Die dominante Bedeutung der Baukosten ist kaum überraschend, ist doch in dieser Kostengrösse vermutlich auch der Bodenpreis repräsentiert, welcher nicht gesondert in die Rechnung aufgenommen wurde.

Einen weiteren entscheidenden Beitrag zur Erklärung der Streunng trugen die Luxusfaktoren Cheminée, Geschirrspüler und hochliegender Backofen bei. In der vorliegenden Rechnung entspricht ihre Gewichtung etwa derjenigen der Wohnfläche, welche seit 1963 pro Einwohner der Stadt um rund 20\% zugenommen hat. Bedeutungsmässig deutlich abgesetzt treten schliesslich noch zwei geographische Standortfaktoren (Lufthygiene und Erreichbarkeit) auf. Bedauerlich ist das Fehlen einer Lärmbelastungsvariable, welche fraglos zur Erklärung der Mietzinsstreuungen beigetragen hätte.

Somit bleiben die Unweltfaktoren für die untersuchte Stichprobe von geringer Bedeutung, gemessen an den Haus- und Wohnqualitätsfaktoren, welche den Objektwert an sich ausmachen. Die der Arbeit zugrundegelegte Hypothese, wonach qualitative Standortfaktoren keinen Einfluss auf die Mietzinsbildung hätten, muss aber dennoch verworfen werden. Augenfällig vorteilhafte Umweltqualitäten (zum Beispiel gute Besonnung, Lage im Grünen o.a.) werden nämlich offensichtlich mitberücksichtigt. Dies bestätigen die Ergebnisse der Umfrage, sowie zahlreiche Wohnungsannoncen, worin auf qualitative Umweltfaktoren als Standortvorteil hingewiesen wird.

Für gravierende Standortnachteile (etwa starke Emissionen von Strassen, Flugschneisen etc.) konnte dasselbe allerdings nicht nachgewiesen werden. In den Interviews wurde meist darauf verwiesen, dass solche umweltbedingte Störfaktoren schwer zu

Tab. 1: Die wichtigsten mietzinserklärenden Faktoren u. ihr Beitrag zur Erklärung der Wertestreuung

Variable Bezeichnung

$\mathrm{X} 6$

$\mathrm{X} 23$

$\mathrm{X} 15$

$\mathrm{X} 31$

$\times 24$

$\mathrm{X} 12$

$\mathrm{X7}$

$\mathrm{X} 20$

$\mathrm{X} 27$

Cheminée
Baukosten in $\mathrm{Fr}$. je $\mathrm{m}^{3}$ Bauvolumen

Wohnfläche netto in $\mathrm{m}^{2}$

Einstellraum im Haus

Geschirrwaschmaschine

$\mathrm{SO}_{2}$-Belastung der Luft in $\mathrm{t} / \mathrm{km}^{2}$

Erreichbarkeit mit privatem Verkehrsmittel in min.

Abstellraum in der Wohnung

Hochliegender Backofen
Beitrag zur Erklärung der Wertestreuung

$30 \%$
$27 \%$
$7 \%$
$9 \%$
$4 \%$
$4 \%$
$2 \%$
$1 \%$
$2 \%$

$27 \%$

$7 \%$

$4 \%$

$1 \%$

$2 \%$ 


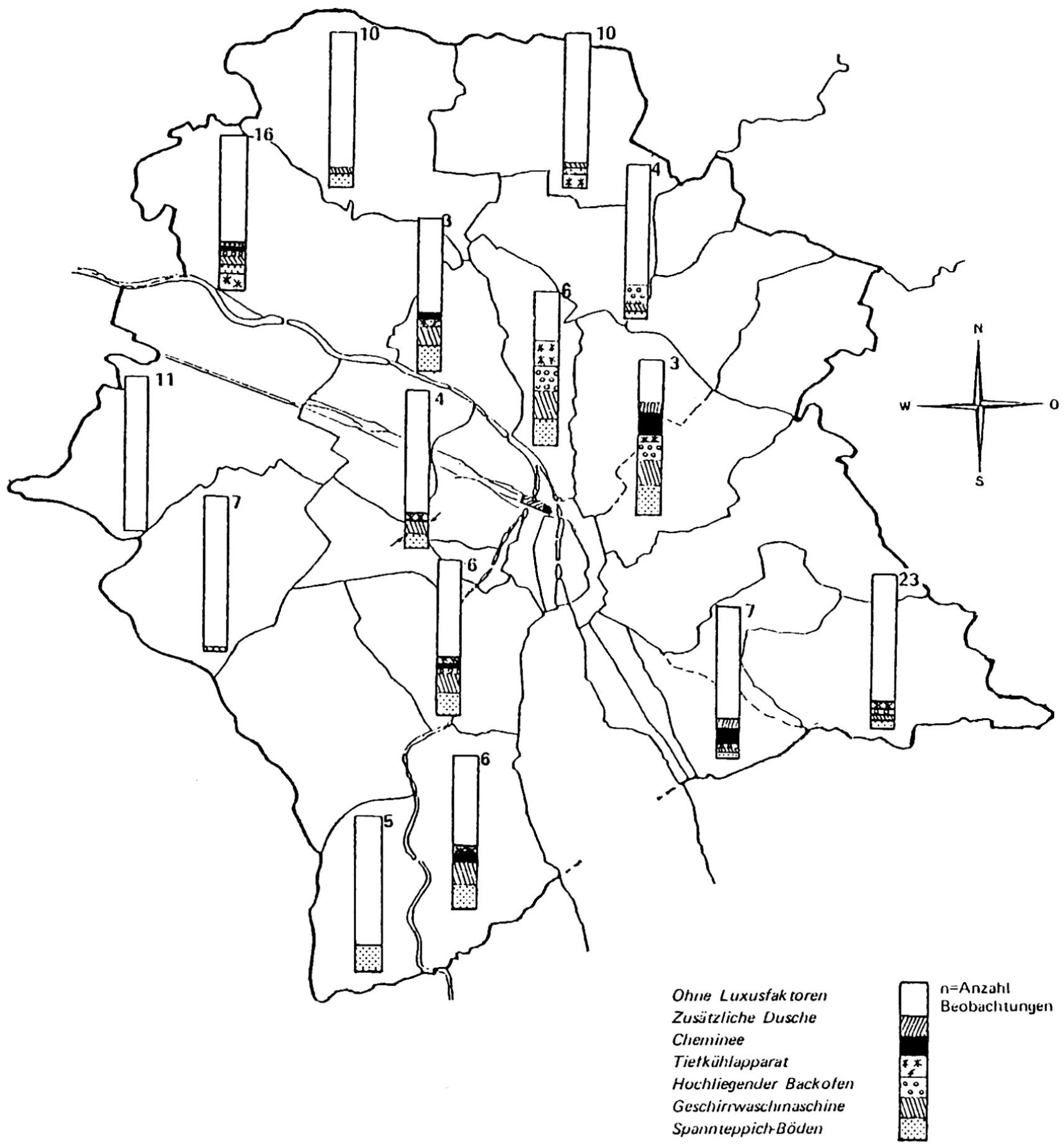

Entwurf: M. Sommer, 1978

Maßstab: $1: 66^{\prime} 500$

$n_{\text {total }}=121$
(Die Säulenhöhe entspricht der Ausstattung alleı uritersuchten Quartieıwohnurigen mit allen 6 Luxusfaktoren. Bei kleinun Beolsachtunyszahlen wurden benachbarte Quarticre zusammen. gefasst.) 
quantifizieren seien und sie so eine ausgewogene Berücksichtigung verunmöglichten.

Das räumliche Verteilungsmuster der Luxusfaktoren in verschiedenen Stadtquartieren (Abb. 3) zeigt.eine Konzentration des Anteils an Luxuswohnungen im Bereich der Stadtquartiere rechts der Limmat sowie in der Enge und in Wollishofen. Aussenquartiere hingegen weisen einen geringeren Anteil an Wohnungen mit Luxusfaktoren auf. Es wird deutlich, dass diese Randquartiere weniger kaufkraftstarke Nachfragerschichten zu versorgen haben.

\section{Schlussbetrachtungen}

Gesamthaft konnte festgestellt werden, dass der materielle innere Wohnwert der neuerstellten Wohnungen gegenüber der vergleichbaren Bausubstanz der 50-er Jahre enorm gesteigert wurde. Vorab vergrösserte sich der Wohnflächenanteil pro Kopf, aber auch die Sanitär- und Haushalteinrichtungen konnten den gewachsenen Wohnbedürfnissen angepasst werden.

Die Mietzinse für die untersuchten Neubauwohnungen werden streng marktwirtschaftlich kalkuliert und sind weitgehend an messbaren Kostengrössen orientiert. Anlageberechnungen und eine dem Investitionsrisiko angepasste, von Fall zu Fall differierende Rendite bilden das Zinsgerippe, welches auf die orts- und quartierüblichen Mietzinse abgestimmt sein muss. Hauptsächlich zinssteigernd sind die Luxusfaktoren, also jene Ausstattungsmerkmale, welche das Wohnniveau über einen mittleren Durchschnitt hinaus anheben (z.B. ein Cheminée).

Der Objektwert einer Wohnung an sich dominiert in städtischen Räumen bei weitem über die positiven oder negativen Wohnumfeld-Kriterien bei der Mietzinsbildung. Dass das Schwergewicht in der Beurteilung des Wohnwerts auf dem Objektwert liegt, deutet auf

- eine verstärkte Mobilität hin, welche es erlaubt, Versorgungs- und Erholungsbedürfnisse standortungebunden zu befriedigen.

- steigende Individualitätsansprüche in städtischen Sozialverhalten hin. Als Folge davon ist mehr und mehr ein Mangel an nachbarschaftlichen Kontakten zu beobachten.
- gestiegene Wohnflächen-Ansprüche hin, welche die immer stärker verdrängte Nächsterholung im Wohnumfeld kompensieren sollen. Städtischer Boden ist allgemein zu teuer, um als Garten- oder Spielraum ausgestaltet $\mathrm{zu}$ werden. Insgesamt haben die 60-er und 70-er Jahre des Zürcher Wohnungsbaus also gezeigt, dass die gestiegenen Wohnbedürfnisse nur teilweise, nämlich im Hausinnenbereich, befriedigt wurden.

Es scheint nach Meinung von Wohnungsvermietern die Devise zu gelten: wie man wohnt ist wichtiger als wo man wohnt.

\section{Literatur}

(1) Aellen, K. et al.: Das Wohnungsbewertungssystem (WBS), Schriftenreihe Wohnungsbau 28d der eidg. Forschungskommission Wohnungsbau, Bern 1975.

(2) Geiger, M.: Die Berechnung von Qualität und Wert von Wohnstandorten, Schriftenreihe Wohnungswesen Bd.10, Bern 1979.

(3) Gesundheits- und Wirtschaftsamt Stadt Zürich: Emissionskataster der Jahre 1972 und 1976, in: Tages Anzeiger der Stadt Zürich, 9.Sept. 1978.

(4) Grandjean, E.: Wohnen im Neubau, eine interdisziplinäre Untersuchung über die Wohnbedingungen in Zürcher Neubauwohnungen und deren Beurteilung durch die Bewohner, Institut für Hygiene und Arbeitsphysiologie der ETH Zürich, Bern 1976.

(5) Gutersohn, H.: Sonnenstrahlung und Bergschatten, in: Vierteljahresschrift der naturforschenden Gesellschaft Zürich LXXIX, Zürich 1934 .

(6) Jenny \& Voorhees, Ing.büro Zürich, und Stadtplanungsamt Zürich: Basismodell der Reisezeiten im privaten und öffentlichen Verkehr, Zürich 1978.

(7) Sommer, M.: Wohnwert und Mietzins, eine quantitative Untersuchung zur Stadtgeographie von Zürich, Dipl.arbeit am Geographischen Institut der Universität Zürich, 1978.

(8) Walti,H.: Die Bestimmungsfaktoren des Mietpreises, Diss. Handelshochschule St.Gallen, 1961 . 